\title{
COMPENSATION FOR PATIENT MOTION IN NUCLEAR MEDICINE RENAL STUDIES BY FAST CORRELATION IMAGE REGISTRATION
}

\author{
M.F. Georgiou, J.H. Nagel, A.V. Cideciyan, G.N. Sfakianakis \\ Departments of Radiology, Biomedical Engineering and Ophthalmology, School of Medicine and College of Engineering, \\ University of Miani, Coral Gables and Miami, Florida, USA
}

\begin{abstract}
A computer method has been developed to compensate for patient motion which degrades nuclear medicine renal dynamic studies. The method is based on a last corrclation algorithm which accomplishes decoupling of the registration variables translation, rotation and scaling, and converts rotations into cyclic shifts using polar transtormations of the images in the Fouricr domain. The method has been implemented into a tully automated program which compensates tor translational and rotational differences between images acquired while the patient was immobile and images acquired after the occurrence of motion. Prcliminary results indicatc the usefulness of the program as a clinical tool for salvaging renal scintigraphic studies with patient motion, thus preventing emoneous interpretations or repeat of the examinations.
\end{abstract}

\section{INTRODUCTION}

Patient motion is a serious problem in nuclear medicine dynamic renal imaging (renal scintigraphy). A dynamic renal scintigraphic

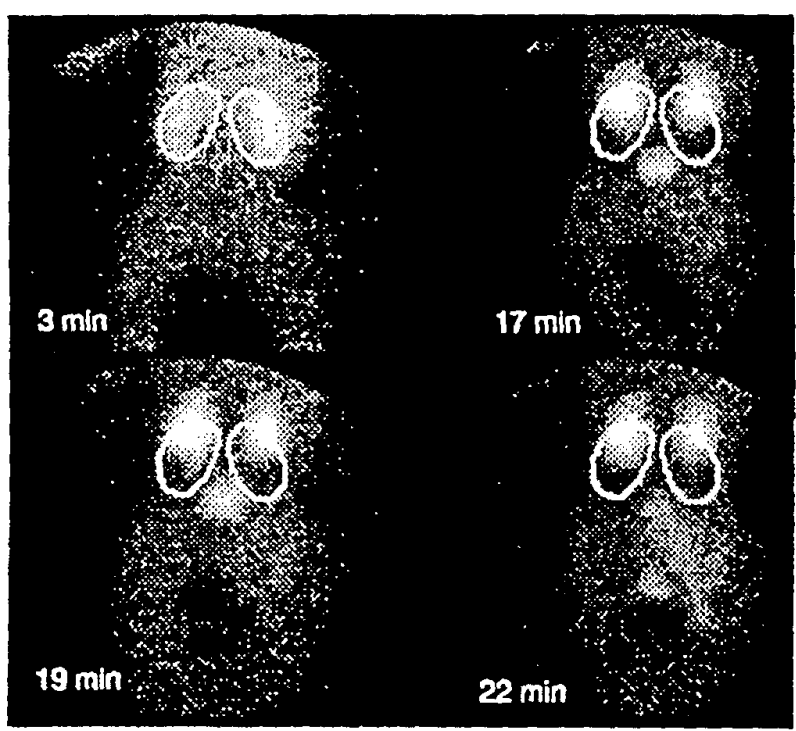

Fig.1: Renal scintigrapinic images with superimposed ROls, showing the effect of patient motion. The ROIs have been selected in the first image of the shown sequence $(3 \mathrm{~min})$, and are applied to the following images without change of their position.

study is acquired by a gamma camera and stored on the computer as a set of sequential planar images (frames), each of $30 \mathrm{sec}$ duration for a total of 22 minutes. The duration of the study makes it very difficult tor the adult patient to remain immobile because of discomfort, back pain, and, occasionally the need to miclurate; children are restricted but, even then, may substantially move. Although visual appreciation of sequential images may not be severely affected by patient motion, quantitative analysis is seriously impaired. As with most nuctear medicine studies, renal function and disease are quantitated by computer analysis which generates time-activily curves (renograms), representing the transit of the radioactivity (radiopharmaceutical) through the kidney(s). To obtain renograms from the sequential images by a semiautomated computer approach it is necessary to assign regions ot interest, i.e. kidney(s) and/or parts thereof, and calculate the radioactive counts within the boundaries assigned in the entire set of acquired sequential images. Each region of interest (ROI) should include the same anatomical site throughout the entire set of images. Patient motion during the acquisition period results in random changes of the anatomical site which is selected on the first or another representative image of the set (Figure 1). The resulting renogram is erratic and does not represent the expected iunction of the selected organ (Figure 2). Furthermore, the effects of motion are not always easily recognizable and thus may result in erroneous interpretations. Conversely, when molion is apprecialed, the entire study may be discarded and a repeat may be decided with all its negative consequences. Theretore, it is understandable that salvaging such studies through methods which compensate for patient motion by registration of the entire image sets would help the patient and the health care protessionals.

\section{MATERIALS AND METHODS}

Image Registration Method: Registration of images is achieved by finding the maximum cross-correlation coetficient in an appropriately transtormed domain. Details of this method and its application to multi-modality (PET, MRI) images $[2,3]$ and high resolution retinal images $[4]$ have already been published. In short, it is assumed that the transformation between any two consecutive (in time) images in the dynamic renal study can be well approximated by a combination of trans/ation and rotation. The determination of the shift parameters and the rotation angle is accomplished in two steps: In the first step, the Fourier magnitude of each image is used to decouple rolation from translation by effectively centering the information content of each image. Next, the Fourier magnitude images are transformed into polar coordinate representation, $r$ and $\Theta$, which converts rotation into a cyclic shitt along the $\Theta$-coordinate. The two-dimensional crosscorrelation function (cyclic in $\Theta$, linear in $r$ ) is calculated for the two polar Fourier magnitude images. The position of the maximum determined along the zero $\mathrm{r}$ shift protile of the crosscorrelation tunction specifies the optimum rotation angle between the images. In the second step, one of the original images is rotated appropriately, and is cross-correlated with the orher original image. The maximum of this cross-correlation function specifies the optimum translation parameters. 
Computer Program: The renal study consists of 44 frames at 30 sec/frame acquired in a $128 \times 128 \times 16$ bits matrix. Typically, patient motion occurs during the later part of the siudy, as the patient gets tired and uncomfortable (Figure 2). Motion could of course occur

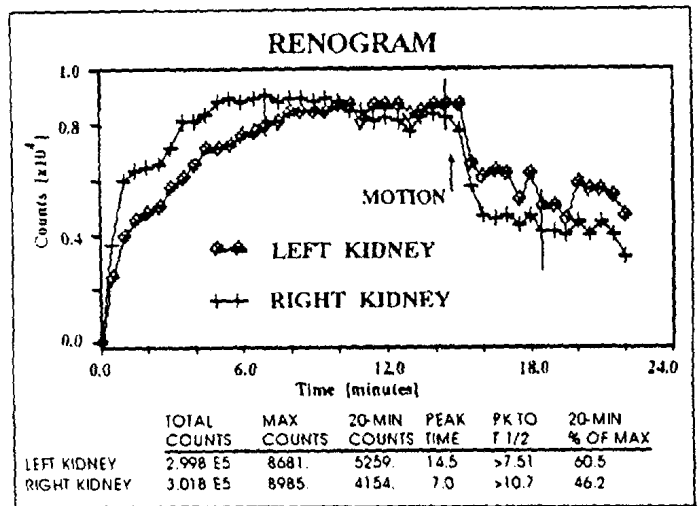

Fig. 2: Frratic renogram, showing a substantial drop in counts as the teffect of patient motion after the $15 \mathrm{sih}$ minute.

at any other time during the study as well. The motion can be detected visually by displaying the sequential images as a dynamic series in cinematic ("cine") mode, or by placing ROls around the kidney(s) on the early trames and applying them to subsequent irames through the end of the study. There are two approaches to selecling a reterence trame tor registration: In the tirst approach, every trame acquired after patient movement is registered with the last irame acquired before patient movement (teterence tramel. Subsequently, the calculated translational and rotational ditferences are compensated for, and the transtormed images are appended to the images without motion. The new image set is then used for ROI placement and renogram generation. In the second approach the lact that the image content (amount of activity inside the kiuncys), changes as the study progresses is taken into consideration in the following way: Each trame with motion is registered with the previous image in the dynamic scries and the relative translation and rotation paramelers are calculated. The registration proceeds backwards in time in a chain fashion, until no rotation and translation changes are tound (or they may be smallet than a maximum allowable (imit) between neighboring trames. At that time, the overall transiational and rolational differences of each frame with motion are computed with respect to the trame without motion and the appropriate transformations lake place. The corrected series is thus available for ROl placement and renogram generation.

\section{RESULTS}

The method was evaluated with ten clinical cases that demonstrated motion and it was found to pertorm equally well for native as well as transplanted kidneys. Figure 3 shows the motioncorrected renogram for the patient whose motion characteristics are illustrated in Figures 1 and 2 . The sudden decrease in counts seen in Fig. 2 atter 15 minutes as a consequence of motion and the mismatch of the ROls disappear. The method presented in this paper yieided objective results in all cases using the single reterence trame approach, even though higher cross-correlation coetticients were derived from the moving reference trame approach.

\section{CONCLUSION}

The described method of compensation for patient motion in renal scinligraphic studies using fast correlation algorithms for image

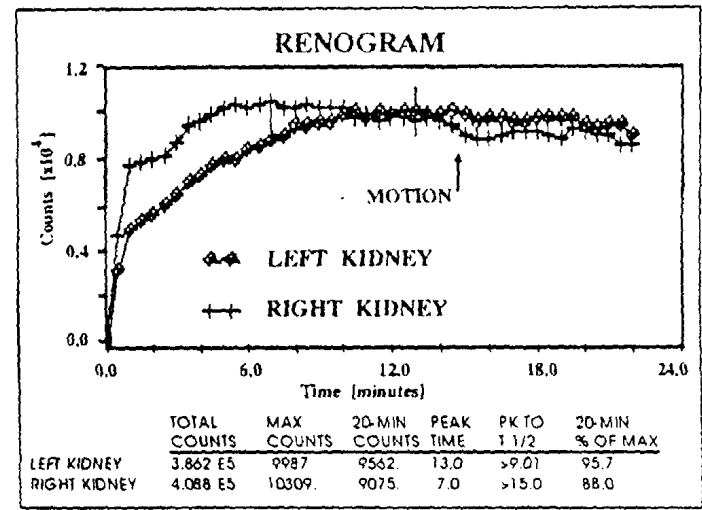

Fkn. 3: Corrected renogram, obloined from the same image study a in Figure 2. but after compensation of patient motion. The renogram shows no more unphysiological decline at the time of the patient motion which is indicated by the arrow.

registralion provides objective results in clinjcal cases with patient movements during data acquisition. It is a uselul clinical too because it can save studies which would otherwise need to be repeated, thus eliminating additional costs in labor, ime and materials. The performance of the method suggests that the registration algorithms may be applied in other planar acquisitions for correction of patient motion, such as liver transplant quantitation, and with appropriate modifications it could also be applied to tomographic siudies, such as cardiac SPECT

\section{REFERENCES}

(1) G.N. Stakianakis, K. Vonorta, G. Zilleruelo, D. Jaffe, M Georgiou: "Scintigraphy in Acquired Renal Disorders", Nuclear Medicine Annual 1992, Raven Press, Lid., New York. pp. $157.224,1992$

[2] A. Apicella, J.S. Kippenhan, J.H. Nagel, "Fast multi-modality image malching". Proc. SPIE Vol. 1092, Medical Jmaging III: Image Processing, pp. 252.263, 1989.

(3) A. Apicella. "Image matching by correlation analysis. biomedical applications", Ph.D. dissertation, University of Miami, Coral Gabies, FL 1989

[4] A.V. Cideciyan, S.G. Jacubsun, C.M. Kemp, R.W. Knighton, 3.H. Nagel, "Registration of high resolution images of the relina". SPIE. Vol. 1652. Medical Imaging VI: Image processing, pp. 310-322, 1992.

Mailing adodress: Mike F. Georgiou

Division of Nuclear Medicine (C.266)

University of Miami School of Medicine

1611 N.W. 12th Avenue

Miami, FL 33136, USA

email: mgeorgioßumednet miami.edu 